
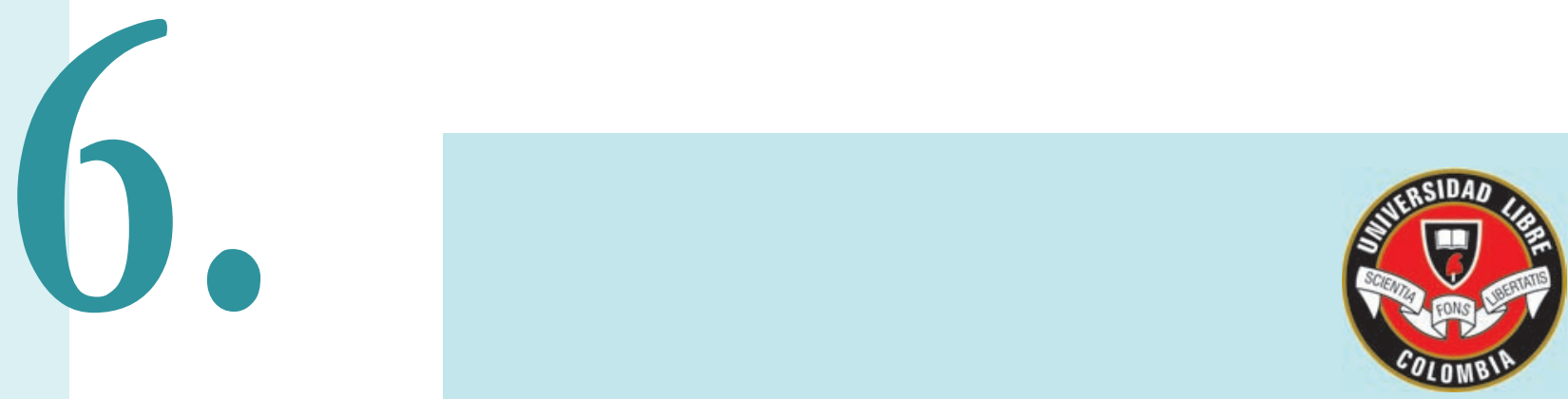

La Calidad Académica, un Compromiso Institucional

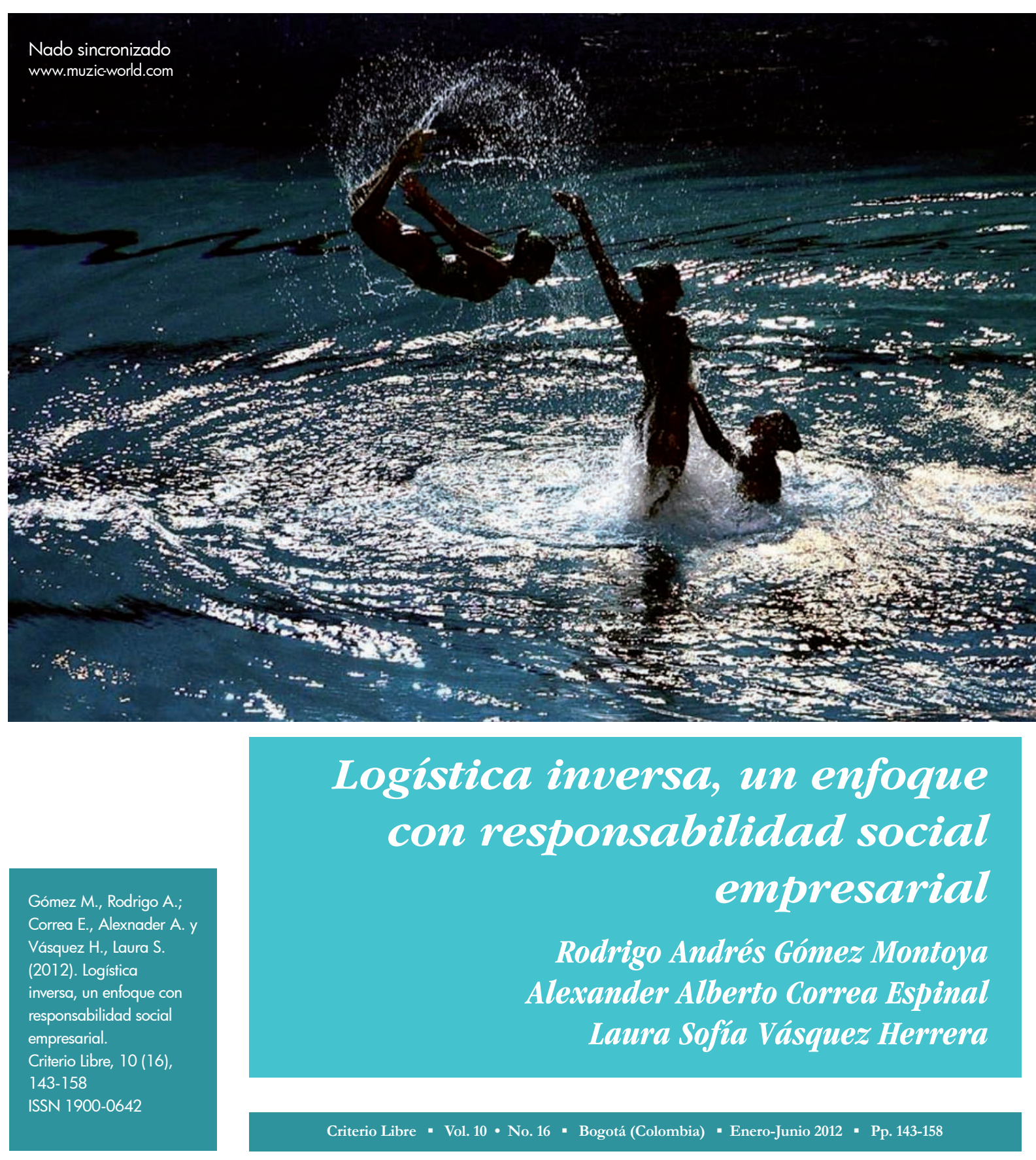




\section{LOGÍSTICA INVERSA, UN ENFOQUE CON RESPONSABILIDAD SOCIAL EMPRESARIAL*}

\section{RODRIGO ANDRÉS GÓMEZ MONTOYA** /† ALEXANDER ALBERTO CORREA ESPINAL $L^{* * *} / \mathrm{t \dagger \dagger}$ LAURA SOFÍA VÁSQUEZ HERRERA ${ }^{* * * *} /+++\dagger$}

Fecha de recepción: septiembre 12 de 2011

Fecha de aceptación: febrero 18 de 2012

Received: September 12, 2011

Accepted: February 18, 2012

Artículo de investigación, producto de la actividad de los docentes en el desarrollo de su línea de investigación.

** Magíster en Ingeniería Administrativa, Universidad Nacional de Colombia; ingeniero industrial, Universidad Nacional de Colombia; profesor, Facultad de Ciencias Agrarias, Politécnico Colombiano Jaime Isaza Cadavid, Colombia; rodrigomezm1986@gmail.com.

*** Ph.D. en estadística e investigación operativa, Universidad Politécnica de Cataluña, España; magíster en Ingeniería Industrial, Universidad de los Andes, Colombia; ingeniero industrial, Universidad Nacional de Colombia; profesor asociado, Escuela de la Organización, Facultad de Minas, Universidad Nacional de Colombia; alcorrea@unal. edu.co.

${ }^{* * * *}$ Ingeniera industrial, Universidad Nacional de Colombia; analista de procesos, El Colombiano S.A., Colombia; laurava@elcolombiano.com.co.

t Research article product of the activity of the professors while developing their research line.

t† Magister in Administrative Engineering, Universidad Nacional de Colombia; Industrial Engineer, Universidad Nacional de Colombia; Professor, Facultad de Ciencias Agrarias, Politécnico Colombiano Jaime Isaza Cadavid, Colombia; rodrigomezm1986@gmail.com.

ttt Ph.D. in Statistics and Operative Research, Universidad Politécnica de Cataluña, España; Magister in Industrial Engineering, Universidad de los Andes, Colombia; Industrial Engineer, Universidad Nacional de Colombia; Associate Professor, Escuela de la Organización, Facultad de Minas, Universidad Nacional de Colombia; alcorrea@unal.edu.co.

t+tt Industrial Engineer, Universidad Nacional de Colombia; Process Analyst, El Colombiano S.A., Colombia; laurava@elcolombiano.com.co.
Criterio Libre $\mathrm{N}^{\circ} 16$ Bogotá (Colombia) Enero-Junio 2012 Pp. 143-158 ISSN 1900-0642 
El presente artículo tiene como objetivo realizar un análisis de la relación entre la logística inversa y la responsabilidad social como estrategia para reducir los impactos en el medio ambiente y aumentar los beneficios sociales y la seguridad en los empleados, clientes y comunidad. Para alcanzar los objetivos, se consideran los temas de logística inversa y responsabilidad social desde la perspectiva teórica, y posteriormente se realiza un estudio exploratorio con empresas del área metropolitana de Antioquia que permita identificar la utilización de este enfoque, importancia e intención de su implementación. Como resultado del artículo, se puede indicar que la utilización de la responsabilidad social en la logística inversa genera grandes beneficios en la cadena de suministro, ya que se desarrollan prácticas amigables con el medio ambiente, aumento potencial de la productividad y la rentabilidad de la empresa y beneficios en la comunidad. Del estudio, se identifica un bajo nivel de utilización de la logística inversa en las empresas con $13 \%$ del total de empresas encuestadas y un interés de $87 \%$ de las empresas en implementarla, debido a los impactos potenciales en la productividad, medio ambiente y beneficios en la comunidad.

\section{PALABRAS CLAVE:}

Logística inversa, Responsabilidad Social Empresarial (RSE), medio ambiente, accionistas.

CLASIFICACIÓN JEL:

M14, M11, Q20.

ABSTRACT

This article's objective is to analyze the relationship between reverse logistics and social responsibility as a strategy to reduce the impact over the environment and increase social benefits and employee, clients and community safety. In order to reach the objectives, the reverse logistics and social responsibility topics were considered from a theoretical perspective and later on an exploratory study is held with companies for Antioquia's Metropolitan area, allowing the use, importance and intention in the implementation of this approach. As a result from this article, it is indicated that the use of social responsibility in reverse logistics generates great benefits in the supply chain because it develops friendly practices with the environment, increasing productivity potential, company profitability and benefits to the community. The study also identifies a low level of usage of reverse logistics in companies with a total of $13 \%$ surveyed companies and an $87 \%$ of companies interested in implementing it due to the potential impact on productivity, environment and benefits to the community.

Key words: Reverse logistics, Social and Corporate Responsibility, Environment, Stakeholders. JEL Classification: M14, M11, Q20. 
presente artigo tem como objetivo realizar uma análise da relação entre a logística inversa e a responsabilidade social como estratégia para reduzir os impactos no meio ambiente e aumentar os beneficios sociais e a segurança nos empregados, clientes e comunidade. Para alcançar os objetivos, consideram-se os temas de logística inversa e responsabilidade social desde a perspectiva teórica, e posteriormente realiza-se um estudo exploratório com empresas da área metropolitana de Antioquia que permita identificar a utilização deste enfoque, importância e intenção de sua implementação. Como resultado do artigo, pode-se indicar que a utilização da responsabilidade social na logística inversa gera grandes benefícios na cadeia de fornecimento, já que se desenvolvem práticas amigáveis com o meio ambiente, aumento potencial da produtividade e a rentabilidade da empresa e benefícios na comunidade. Do estudo, identifica-se um baixo nível de utilização da logística inversa nas empresas com 13\% do total de empresas entrevistadas e um interesse de $87 \%$ das empresas em implementála, devido aos impactos potenciais na produtividade, meio ambiente e benefícios na comunidade.

Palavras chave: Logística inversa, Responsabilidade Social Empresarial (RSE), meio ambiente, acionistas. Classificação JEL: M14, M11, Q20.

RÉSUMÉ

Ce document vise à l'analyse de la relation entre la logistique inverse et la responsabilité sociale comme une stratégie visant à réduire les impacts environnementaux et d'accroître les avantages sociaux et la sécurité des employés, des clients et de la communauté. Pour atteindre ces objectifs, nous considérons les problèmes de logistique inverse et la responsabilité sociale à partir d'un point de vue théorique, puis effectué une étude exploratoire avec des entreprises dans la région métropolitaine d'Antioquia, afin d'identifier I'utilisation de cette approche, l'importance et le but de sa mise en œuvre. Comme résultat de cet article, nous pouvons indiquer que l'utilisation de la responsabilité sociale dans la logistique inverse apporte de grands bénéfices dans la chaîne d'approvisionnement, dans les pratiques de développement respectueux de I'environnement, dans l'augmentation potentielle de la productivité et la rentabilité d'avantages dans la communauté. Cette étude a identifié un faible niveau d'utilisation de la logistique inverse dans les entreprises avec 13\% des entreprises interrogées et un intérêt de $87 \%$ des entreprises dans sa mise en œuvre en raison des impacts potentiels sur la productivité, l'environnement et les avantages dans la communauté.

Mots-clés: logistique inverse, responsabilité sociale des entreprises - RSE, environnement, actionnaires. 
La logística inversa es un proceso dentro de la administración de la cadena de suministro que ha cobrado importancia, ya que permite gestionar los retornos de los clientes impactando en el servicio y recuperación del valor del producto, adecuada disposición final y desarrollo de prácticas amigables con el medio ambiente.

La logística inversa puede considerarse un proceso o elemento crítico para el desarrollo de prácticas de responsabilidad social de las empresas, de allí la importancia e interés de la planificación, ejecución y control de este tipo de sistemas logísticos en las organizaciones y la cadena de suministro, incluyendo relaciones con proveedores y clientes.

Por los motivos expuestos, el presente artículo tiene como objetivo revisar y analizar la relación e impacto de la logística inversa como elemento de la responsabilidad social de las empresas. Los destinatarios del artículo son profesionales, estudiantes e investigadores interesados en conocer acerca de la logística inversa desde la perspectiva de prácticas responsables.

La metodología utilizada para la construcción del artículo se basa en la revisión bibliográfica de libros, revistas científicas internacionales que incluyen autores como Terrado (2007), Mentzer (2004), Murphy (2002), Carroll (2008), los cuales se convierten en la base para relacionar y analizar la logística inversa y la responsabilidad social. El documento se estructuró en los siguientes numerales: logística inversa en la cadena de suministro, logística inversa y Responsabilidad Social Empresarial (RSE) y conclusiones.
En los últimos cinco años el concepto de logística inversa ha cobrado importancia en el sector empresarial, debido a que el enfoque de las organizaciones pretende entregar al mercado productos amigables con el medio ambiente y sostenibles.

En la cadena de suministro que se conforma generalmente por proveedores, empresas transformadoras o prestadoras de servicios, clientes y stakeholders, es de interés desarrollar prácticas de responsabilidad social que generen impactos en el bienestar del personal, los empleados, los clientes, el medio ambiente y la rentabilidad económica buscando una gestión integral.

Por lo anterior, a continuación se presentan los conceptos de cadena de suministro, logística inversa y responsabilidad social empresarial
(RSE), antes de realizar el análisis de un enfoque integrado de los conceptos.

\subsection{CADENA DE SUMINISTRO Y LOGÍSTICA}

Para Terrado (2007), "La cadena de suministro es un enfoque integrado de los procesos logísticos de aprovisionamiento o logística de entrada, fabricación o logística interna, y distribución o logística de salida, permitiendo el desarrollo de las relaciones con los proveedores, la empresa y los clientes". Por su parte, Mentzer (2004) define la cadena de suministro como un conjunto de tres o más empresas conectadas o relacionadas con flujos de productos, servicios, finanzas e/o información desde el aprovisionamiento hasta el cliente final. A continuación se presenta un esquema tipo de cadena de suministro (ver Figura 1). 


\section{Flujo de información, productos y dinero}

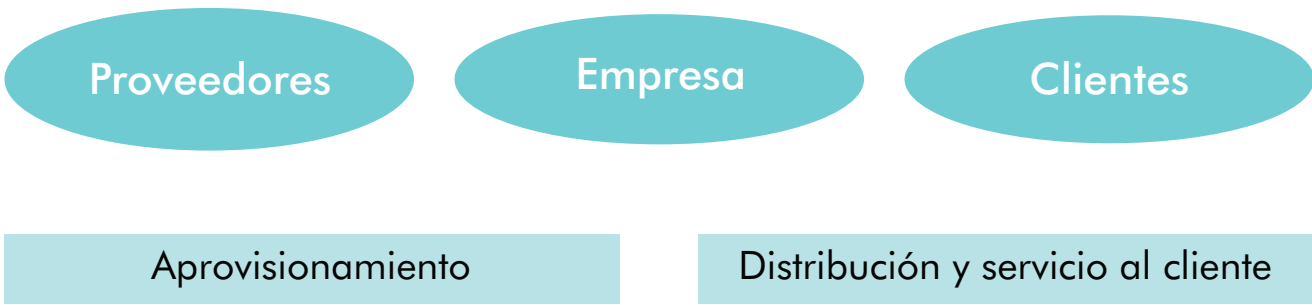

\section{Producción}

Transporte

\section{Almacenamiento}

Logística Inversa

Fuente: Elaboración propia.

A partir de las definiciones y la estructura general de la cadena de suministro (ver Figura 1), se puede indicar que ésta permite desarrollar los flujos de información, productos y dinero desde el aprovisionamiento a los proveedores pasando por la transformación hasta la entrega a los clientes. Además, debe considerarse que el funcionamiento de ésta depende de la planeación y control de un conjunto de procesos logísticos, en los cuales se incluye la logística inversa que es transversal en la cadena de suministro.

\subsection{LOGÍSTICA INVERSA}

La logística inversa comprende el flujo de productos, información y dinero desde el punto de uso hasta el de origen o reproceso, siendo contrario a la dirección tradicional de la cadena de suministro que comprende desde el punto de origen (empresaproveedor) hasta el punto final (distribuidores- clientes). Otro enfoque de la logística inversa es considerarla como un conjunto de procesos encargados de recibir, evaluar, registrar y transformar o tratar los productos retornados por los clientes, para reutilizarlos en el medio industrial o disponerlos adecuadamente para reducir los impactos en el medio ambiente, la comunidad y generar beneficios económicos (Barker y Zabinsky, 2008).

En el ámbito empresarial la logística inversa tiene como objetivo planear, ejecutar y controlar los flujos de productos, información y dinero, mediante la identificación y el diseño de procesos eficientes que permitan su reúso, recuperación, reciclaje o eliminación, con el fin de minimizar los impactos ambientales y maximizar los beneficios económicos de la empresa (Lin, Lee y Lee, 2009). A continuación se presenta el ciclo de vida de la logística inversa, que soporta el cumplimiento su objetivo (ver Figura 2). 


\section{Figura 2. Ciclo de la logística inversa.}

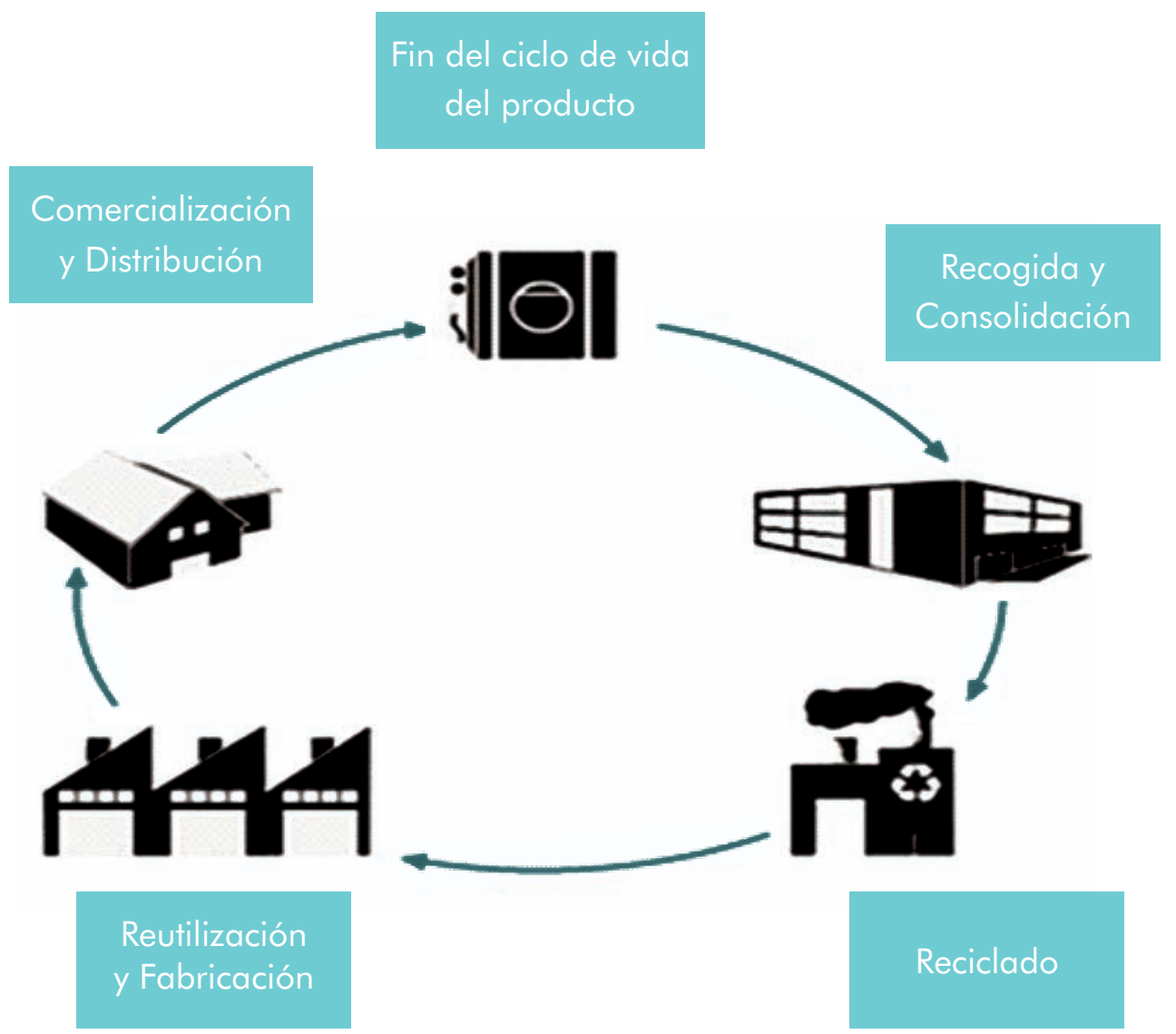

Fuente: Vicarli, 2009.

El ciclo de la logística inversa presenta las diferentes etapas que los productos siguen desde que cumplen un ciclo de vida o no cumplen con las especificaciones de los clientes hasta que son reciclados, reusados, remanufacturados o dispuestos en condiciones adecuadas buscando reducir los impactos ambientales, eficiencia en la utilización de los recursos y recuperación del valor económico.

En el ciclo de la logística inversa se soporta en un conjunto que permite cumplir el objetivo y reducir los impactos negativos en el medio ambiente y la comunidad. Los procesos más comunes son: (1) Recolección, (2) Inspección y selección, (3) Recuperación directa del producto, (4) Transformación o tratamiento final, (5) Transporte y (6) Almacenamiento. (Dyckhoff, Lackes, Reese, 2003; Vellojín, Meza, Amaya, 2008; ver Tabla 1).

A partir de los procesos presentados (ver Tabla 1), se puede indicar que la logística inversa busca realizar la gestión de recuperación de aquellos productos que no cumplen con las expectativas del clientes o aquellos que cumplieron su ciclo de vida y necesitan ser tratados con el fin de evitar daño ambiental, darles un uso alternativo o realizarse una disposición adecuada, como se indicó con anterioridad. 
Tabla 1. Procesos de la logística inversa.

\begin{tabular}{ll}
\hline Recolección & $\begin{array}{l}\text { Recogida de los productos o residuos desde los lugares de uso (cliente) al punto de } \\
\text { origen o recuperación. }\end{array}$ \\
\hline $\begin{array}{l}\text { Una vez los productos son recolectados, se realiza una inspección de los productos o } \\
\text { materiales (empaques) con el fin de determinar la cantidad, procedencia, razones de } \\
\text { devolución y tipo de productos. }\end{array}$ \\
\hline $\begin{array}{l}\text { Inspección y selección } \\
\text { producto }\end{array}$ & $\begin{array}{l}\text { Se realiza cuando el producto recuperado puede ser fácilmente devuelto al mercado o } \\
\text { proceso productivo. }\end{array}$ \\
\hline $\begin{array}{l}\text { Transformación o } \\
\text { tratamiento final }\end{array}$ & $\begin{array}{l}\text { Se encarga de transformar o tratar los bienes o residuos recuperados en productos } \\
\text { reusables o remanufacturados para el uso industrial o convertirlos a un estado amigable } \\
\text { con el medio ambiente. }\end{array}$ \\
\hline Transporte & $\begin{array}{l}\text { Consiste en mover los productos o residuos entre los puntos de uso y origen o } \\
\text { transformación. }\end{array}$ \\
\hline Almacenamiento & $\begin{array}{l}\text { Utilizado para almacenar los productos, materiales o residuos en forma temporal o por } \\
\text { períodos de tiempo programados y controlados. }\end{array}$
\end{tabular}

Fuente: Elaboración propia.

\section{RESPONSABILIDAD SOCIAL Y LOGÍSTICA INVERSA}

La Responsabilidad Social Empresarial (RSE) ha adquirido importancia en la gestión de las empresas, el sistema logístico y la cadena de suministro, debido a las exigencias del mercado y a las regulaciones legales, que en ocasiones las obligan a desarrollar prácticas que sean socialmente responsables y amigables con el medio ambiente y que contribuyan al aumento del bienestar y garanticen la seguridad de los empleados, proveedores, clientes y la comunidad en que opera la organización (Ciliberti, Potrandolfo, Scozzi, 2008). Por estos motivos en el presente numeral se definirá el concepto de responsabilidad social empresarial y también la logística inversa.

\subsection{RESPONSABILIDAD SOCIAL EMPRESARIAL (RSE)}

La responsabilidad social empresarial (RSE) puede definirse como un conjunto de prácticas que buscan generar beneficios sociales, éticos, ambientales y económicos de manera coordinada para impactar en los clientes, empleados, accionistas y la comunidad (Murphy, 2002).
De otra parte, la RSE puede considerarse como una estrategia de negocio que busca generar valor y bienestar a la comunidad y a las partes relacionadas con la empresa, buscando desarrollar una ventaja económica que impacte en el crecimiento y sostenibilidad de la misma, lo cual involucra diferentes procesos de la organización como: logística, producción, finanzas, mercadeo social, entre otros (Carroll, 1999), (Ciliberti, Potrandolfo, Scozzi, 2008).

\subsection{ANÁLISIS DE LA LOGÍSTICA INVERSA COMO PRÁCTICA DE RESPONSABILIDAD SOCIAL EMPRESARIAL (RSE)}

Como ya se indicó, la logística inversa busca gestionar los flujos de productos e información desde los puntos de uso o consumo hasta el origen o transformación, buscando reducir los impactos en el medio ambiente o la comunidad y recuperar el valor económico a los productos. Por estas razones cobra interés realizar un análisis de cómo orientar las estrategias y actividades de la logística inversa como prácticas socialmente responsables que 
permitan a la organización generar una ventaja competitiva sostenible y orientada a beneficiar a las partes interesadas en la cadena de suministro. Por ello, a continuación se realiza un análisis de cómo apropiar los principios de RSE en la logística inversa basándose en autores como Carroll, 1999; Ciliberti, Potrandolfo, Scozzi, 2008; Carter y Jennings, 2002; Carter y Rogers, 2008 (ver Tabla 2).

\section{Tabla 2. Logística inversa y RSE en el ámbito empresarial.}

\section{1) Enfoque general del RSE en la logística inversa}

- Planeación, diseño, implementación y control de procesos de reúso, remanufactura, reciclaje o disposición final que reduzcan el impacto en el medio ambiente y beneficien a la comunidad.

- Se deben establecer estrategias de logística inversa que cubran la cadena de suministro como: proveedores, productores, distribuidores, cliente final, empleados, accionistas y comunidad.

- Las empresas que utilizan la RSE pueden aumentar la participación en el mercado desarrollando un posicionamiento con prácticas como: producción más limpia, conservadores del medio ambiente y justos socialmente con los stakeholders [16].

- RSE en la logística inversa puede basarse en una metodología, que incluye etapas como: a) identificación y análisis de los riesgos de impactos ambientales o sociales que causen posibles daños, perjuicios o beneficios en el desarrollo de la RSE en los procesos de logística inversa, b) Diseño, implementación y control de políticas y procedimientos de RSE que las maximicen los beneficios o minimicen los riesgos para los clientes, la comunidad, el medio ambiente y/u otros actores, c) Alineación de las estrategias y prácticas de RSE con la utilización de una norma internacional como la AS 8000, ISO 26000, ISO 14001, entre otras que orientación de la RSE en la logística inversa.

\section{2) Apropiación de los principios de RSE en la logística inversa}

\section{Medio ambiente}

- Diseñar e implementar procesos logísticos amigables con el medio ambiente que permitan reducir el consumo de materias primas, emisión de $\mathrm{CO}_{2}$, entre otros.

- Utilizar prácticas de producción más limpia u orgánica que promueva la protección y cuidado del medio ambiente.

- Promover políticas medioambientales en la negociación a nivel de proveedores, productores, distribuidores, minoristas y clientes finales, etc.

- Diseñar planes de logística inversa que consideren los tratamientos o posibles usos de los productos en las diferentes etapas del ciclo de vida que permita reducir los impactos en el medio ambiente y aumentar la recuperación del valor del producto.

- Un ejemplo es definir en qué condiciones realizar un reúso, una remanufactura, reciclaje y cómo desarrollar la disposición de los productos, en caso que sea necesario.

\section{Seguridad}

- Promover un ambiente seguro para cada empleado dentro de los procesos de logística inversa.

- Esto cobra importancia ya que en los procesos de reciclaje, reúso y recuperación del producto los empleados suelen realizar procesos con químicos que pueden afectar la salud y el bienestar de las personas.

- Fomentar el desarrollo y promoción de prácticas de seguridad en su empresa y la cadena de suministro.

\section{Códigos y normas}

- Certificar las prácticas de RSE en la logística inversa a través de la implementación de normas internacionales, en especial la ISO 14001 que permite demostrar que la empresa garantiza una operación amigable con el medio ambiente y la comunidad.

- Se debe considerar la AS 8000 y la ISO 26000 como prácticas de RSE integrales.

- Existe normativa como los sellos de producción orgánica o normativa sectorial o legal relacionada con reciclaje que permite demostrar que la compañía cumple con las prácticas RSE.

\section{Responsabilidad financiera}

- Desarrollar estudios de costos y rentabilidad de los diferentes procesos de la logística inversa.

- Incluir en los estudios impactos económicos de los procesos logísticos en el medio ambiente y la comunidad.

- Realizar acuerdos financieros transparentes que no afecten al actor con que se realice la negociación, ni al gobierno ni a la comunidad en general. 


\section{Tabla 2. Logística inversa y RSE en el ámbito empresarial (cont.).}

\section{2) Apropiación de los principios de RSE en la logística inversa}

\section{Derechos humanos y ética}

- Acatar y cumplir con las condiciones de las alianzas con los socios comerciales, la normatividad legal y los tratos establecidos con otros actores que se relacionan con la empresa.

- No desarrollar procesos empresariales o emplear insumos que afecten la salud y bienestar de los empleados, la comunidad y los clientes.

- Respetar el código de conducta de la empresa y la cadena de suministro.

- Tratar a los empleados con respeto y dignidad, y no utilizar menores en condiciones de explotación laboral en los diferentes procesos de la logística inversa.

- Fomentar el respeto a los derechos humanos dentro de la empresa y su cadena de suministro.

\section{Comunidad}

- Desarrollo de los diferentes procesos de logística inversa como reúso, reciclaje y disposición final de los productos que no generen impactos negativos en la salud y bienestar de las personas de la comunidad.

- Generar beneficios económicos, sociales y sostenibles ambientalmente en el entorno de las comunidades en que opera la empresa. Un ejemplo de esta situación son los planes ambientales y reciclaje.

- Fomentar e implementar prácticas de RSE en las empresas que mejoren las condiciones laborales, desarrollo económico y fomento de la industria relacionada con la empresa buscando una participación activa de la comunidad.

- Desarrollar campañas sociales y reinversión en la comunidad que impacte en las personas y sostenibilidad de la organización.

Fuente: Elaboración propia.

Del análisis de la relación, se puede indicar que la logística inversa busca gestionar los retornos, procesos de remanufactura, reproceso, reciclaje y disposición de los productos, buscando reducir los impactos en el medio ambiente y aumentar la recuperación del valor económico de los productos. Con la utilización de algunos principios de la RSE, la logística inversa puede aumentar el alcance de los impactos en la cadena de suministro, ya que no sólo se genera reducción en los impactos medioambientales sino que también se establecen beneficios para los empleados, la comunidad y los clientes en aspectos como ética, derechos humanos, seguridad y salud y responsabilidad financiera. Por tal razón, se puede indicar que la logística inversa y la RSE pueden considerarse como una estrategia que puede impactar en el crecimiento, sostenibilidad y una gestión integral de la empresa que trascienda lo económico y la productividad para considerar aspectos sociales.

\section{3. metodología}

Con el fin de describir y analizar la importancia, uso y proyección de la implementación de la logística inversa como práctica de responsabilidad social, se realiza un estudio basado en la utilización de una encuesta aplicada a seis empresas de diferentes tamaños y sectores empresariales ubicadas en el área metropolitana del departamento de Antioquia.
Para la realización del estudio se utilizan cuatro etapas, que se describen a continuación (ver Figura 3).

La primera etapa de planeación del estudio y diseño de la encuesta permite establecer el objetivo, que consiste en desarrollar exploración inicial y contextualización de la 
importancia, implementación o intención de uso de la logística inversa como práctica de responsabilidad social en 12 empresas localizadas en el área metropolitana. La ficha técnica del estudio exploratorio está basada en encuestas (ver Tabla 3).

Figura 3. Metodología de estudio de la logística inversa como práctica de responsabilidad social.

\section{Ełapa 1. Planeación y diseño del \\ estudio, incluyendo la encuesta}

Etapa 3. Procesamiento y análisis de la información del estudio.
Etapa 2. Recolección de los datos de las empresas.

Etapa 4. Conclusiones y recomendaciones para establecer importancia, uso y proyección de la logística inversa.

Fuente: Elaboración propia.

\section{Tabla 3. Ficha del estudio y encuesta para logística inversa como práctica de responsabilidad social.}

Herramienta de recolección

Variables del estudio
Se utiliza una encuesta con seis preguntas cerradas y abiertas relacionadas con las variables de interés en la exploración y contextualización.

El sector empresarial, tamaño de la empresa, importancia de la logística inversa como práctica de responsabilidad social, el uso actual de la logística inversa en la compañía, y cómo proyecta o tienen interés en su implementación en caso de que no se utilice actualmente, o cómo debe mejorarse si actualmente utiliza la práctica, y que norma de responsabilidad social utiliza o implementaría para apoyar la práctica.

\section{Características y tamaño la muestra}

Se seleccionan empresas manufactureras, comercial y servicios de diferentes tamaños y sectores, que permitan realizar una exploración y contextualización de la logística inversa como práctica de responsabilidad social.

Como muestra para la exploración y contextualización, se seleccionan empresas del área metropolitana de Antioquia de los sectores de manufactura, comercial y servicios; Las empresas se identificaron en bases de datos de la cámara de comercio de Medellín, Aburra Sur, y directorios comerciales. Por ser un estudio de exploración, se seleccionan 12 empresas, cuyo sector, actividad y tamaño se describe a continuación.

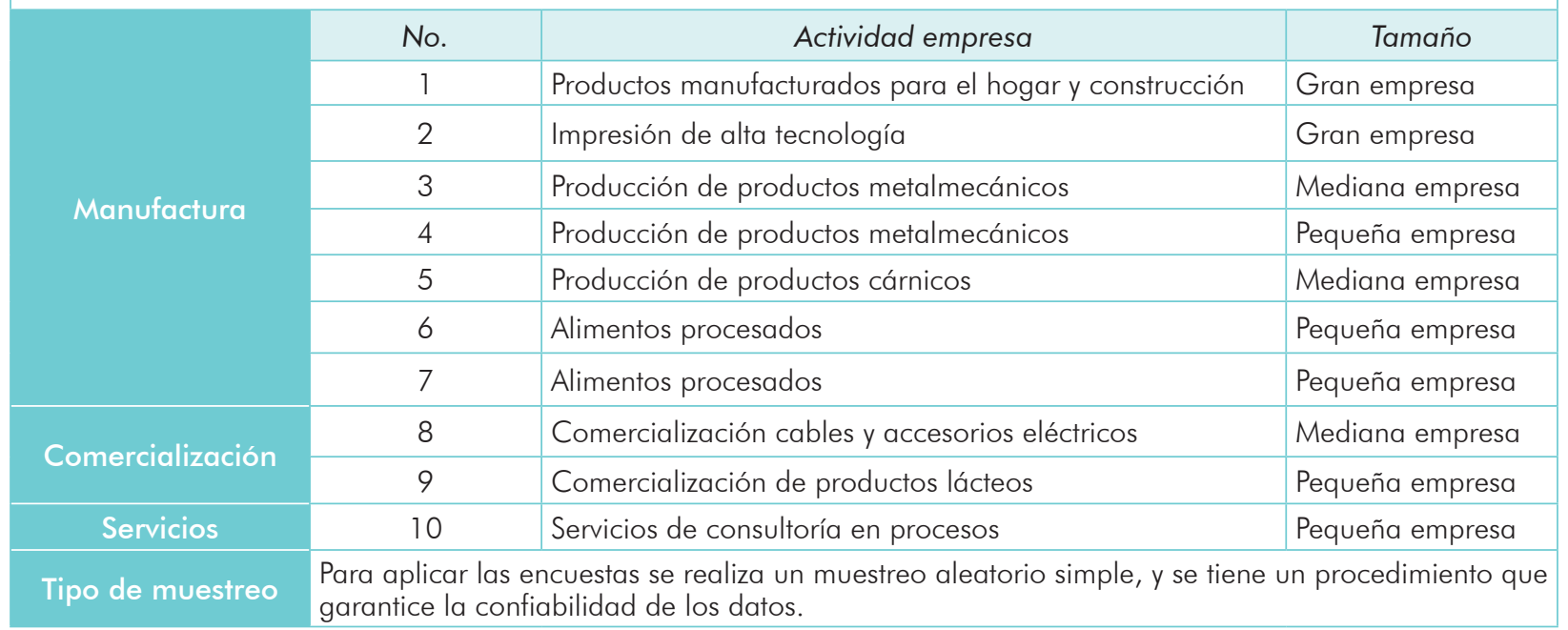

Fuente: Elaboración propia. 
En la segunda etapa, se recolectan los datos que consisten en la aplicación de la encuesta a través de la entrevista de administradores, directores de logística de las empresas o personal relacionado con responsabilidad social empresarial.

En la tercera etapa se realiza el procesamiento, que consiste en organizar los datos de las variables en estudio clasificándolas por tamaño y sector económico de la empresa. Posteriormente se realiza un análisis de los datos gráficos de participación, diagrama de Pareto, entre otros, que permitan estudiar la importancia, nivel de uso y proyección de la utilización del estudio.

En la cuarta etapa se presentan conclusiones y recomendaciones que permiten establecer la importancia, uso y proyección del uso de la logística inversa de responsabilidad social. Además, se establecen los trabajos de investigación futura.

\section{RESULTADOS Y DISCUSIÓN}

Una vez realizadas las seis encuestas en las seis empresas seleccionadas en el estudio, se analizan los datos de variables como: utilización de la logística inversa como práctica de responsabilidad social, importancia de la logística inversa en el desarrollo de la estrategia de la compañía, proyección de implementación o mejoramiento de la logística inversa con enfoque en la responsabilidad social.

Utilización logística inversa como práctica de responsabilidad social. Para esta variable se obtienen los siguientes resultados (ver Figura 4).

Figura 4. Utilización de logística inversa como práctica de responsabilidad social.

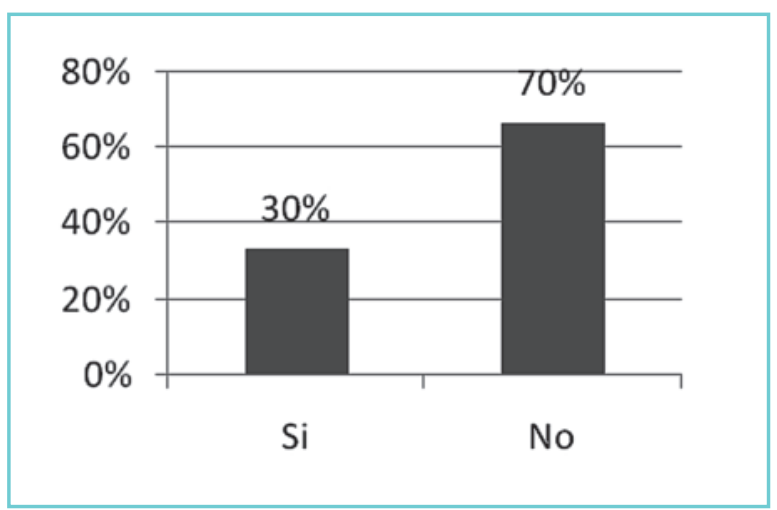

Fuente: Elaboración propia.
De los resultados, se puede indicar que la logística inversa es una práctica con bajo nivel de implementación en las empresas, como se representa en la exploración ya que sólo $30 \%$ de las empresas la utilizan, en especial las grandes. Una de las razones detectadas del bajo nivel de implementación es el desconocimiento de las empresas acerca de la logística inversa, la capacidad de inversión y el conocimientos de los impactos económicos, ambientales y relaciones con los accionistas de la empresa que su uso puede traer.

Importancia de la logística inversa. Para cada una de las empresas se evalúa: a) la importancia de la logística inversa como práctica de responsabilidad social que impacta la sostenibilidad de la compañía, y b) los factores que hacen importante la logística inversa en las empresas.

Para responder a esta pregunta se realizó una explicación de las características, importancia e impactos de implementar la logística inversa como práctica de responsabilidad social (ver Figuras 5 y Figura 6).

Aproximadamente $80 \%$ de las empresas manifiestan la importancia de la implementación o desarrollo de estrategias relacionadas con la implementación de un sistema de logística inversa como práctica 
de responsabilidad social por el impacto potencial en la productividad, servicio al cliente, cuidado del medio ambiente y relaciones con la comunidad. La única empresa de las seis que responde que no considera importante ni prioritario este enfoque lo hace debido a su tamaño, característica de la empresa por estar dedicada a los servicios e inversiones en estrategias de gestión.

Figura 5. Importancia de implementar sistema de logística inversa como práctica de responsabilidad social.

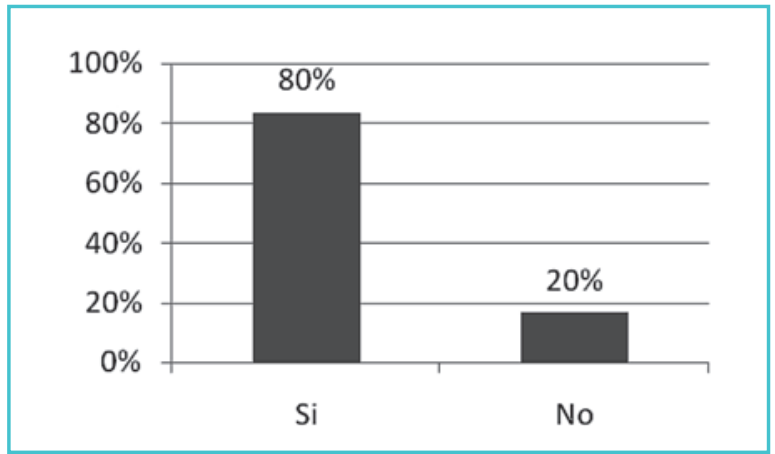

Fuente: Elaboración propia.

Figura 6. Factores de interés de la logística inversa y responsabilidad social.

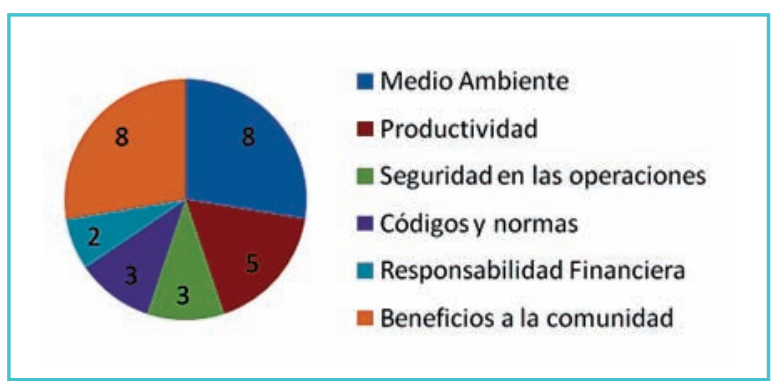

Fuente: Elaboración propia.

De los diferentes factores, la contribución al medio ambiente, los beneficios a la comunidad y la productividad son los más significativos para las empresas, si adoptaran un enfoque de logística inversa como práctica de responsabilidad social. Estos factores deben considerarse de interés para promover programas que motiven a las empresas para su implementación. De otra parte,
${ }^{6}$ De los diferentes factores, la contribución al medio ambiente, los beneficios a la comunidad y la productividad son los más significativos para las empresas, si adoptaran un enfoque de logística inversa como práctica de responsabilidad social. Estos factores deben considerarse de interés para promover programas que motiven a las empresas para su implementación. 
${ }^{66}$ La logística inversa es un proceso dentro de la cadena de suministro que permite establecer estrategias para gestionar adecuadamente los retornos, el reciclaje, la remanufactura o disposición de los productos; de allí la importancia de una adecuada gestión de este proceso debido a que influye en la reducción de impactos ambientales $y$ recuperación del valor económico. puede considerarse que el medio ambiente y los beneficios a la comunidad son factores reconocidos en el ámbito empresarial en programas de responsabilidad social. Además de la exploración, se identifica la oportunidad de promover estudio, investigaciones y programas que muestren el impacto de la logística inversa como práctica de responsabilidad social en factores como la productividad y la rentabilidad financiera.

Intención de implementación o mejoramiento de logística inversa como práctica de responsabilidad social. Se obtienen los siguientes resultados considerando la justificación realizada por la empresa (ver Figura 7).

Figura 7. Intención de mejoramiento o implementación de logística inversa como práctica de responsabilidad social.

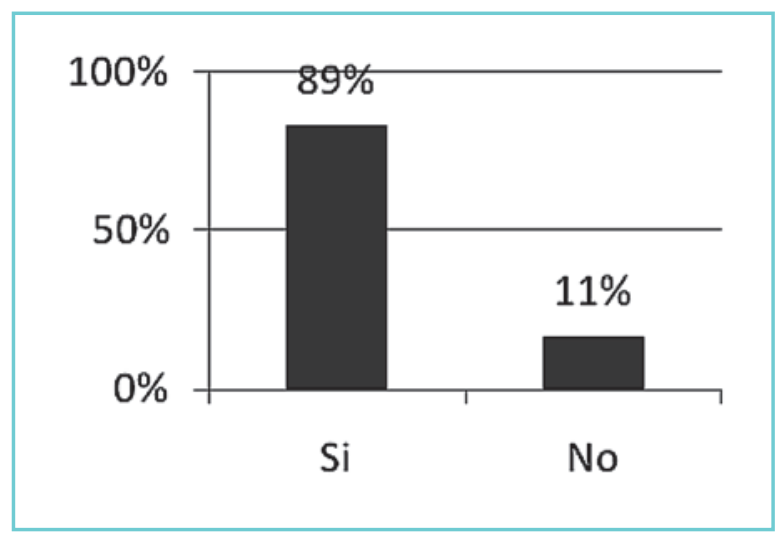

Fuente: Elaboración propia.

Del $89 \%$ que responde afirmativamente, tres de las empresas que tienen implementada logística inversa como práctica de responsabilidad social indican interés en mejorar su alcance incluyendo estrategias que permitan aumentar la productividad, medición de indicadores financieros e impactos en la comunidad desarrollando actividades empresariales, debido a que cuentan con programas de reciclaje y venta de productos clasificados como de calidad tipo II, que son funcionales pero con defectos en los empaques. Las otras empresas manifiestan interés a mediano plazo (mayor que 
un año) de implementar prácticas de logística inversa como práctica de responsabilidad, en especial, procesos de reciclaje, remanufactura y disposición de desechos planificados buscando mejorar la relación con la comunidad, el medio ambiente y la productividad, considerado como crítico para la sostenibilidad de la empresa. Cabe recordar que una empresa que se dedica a la prestación de servicios, indicó que no le interesa implementar procesos de logística inversa como práctica de responsabilidad social debido a las características de la empresa, tamaño y capacidad de inversión.

Respecto al uso de los estándares que se utilizarían de referencia para implementar la responsabilidad social y la logística inversa (ver Figura 8), 56\% utilizaría el AS 8000 que es el más reconocido; es decir, $56 \%$ de las empresas emplearía el estándar colombiano de responsabilidad social ISO 26000 y otros prácticas propias. Debe señalarse que las dos empresas que tienen implementados procesos de logística inversa con responsabilidad social, tienen certificado de la AS 8000, alineado con prácticas del sistema logístico de la empresa.

De los diferentes resultados obtenidos del estudio de la utilización de la logística inversa como práctica de responsabilidad social, se identifica el bajo nivel de implementación y conocimiento de sus características e impactos potencias en el beneficio a la comunidad, la productividad, el cuidado del medio ambiente, la responsabilidad financiera, los cuales no sólo impactan en la sostenibilidad de la empresa sino también las relaciones con los actores o stakeholders de la empresa.
Figura 8. Estándar que se utilizaría para responsabilidad social y logística.

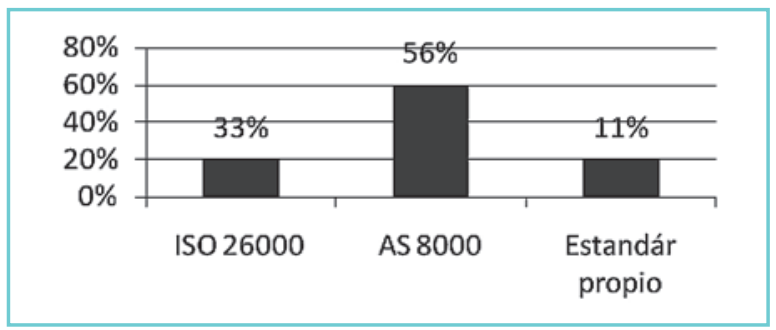

Fuente: Elaboración propia.

De otra parte, se identifica el potencial del uso de la logística inversa como práctica de responsabilidad social, ya que de las empresas encuestadas en el estudio, un $80 \%$ se identifica con este uso, después de conocer los beneficios e impactos que puede obtener con la implementación de este enfoque, ya que la logística inversa permite desarrollar procesos que pueden ser planificados y controlados, como reciclaje, reúso, remanufactura y disposición final y pueden orientarse al aumento de la productividad, rentabilidad financiera, amigabilidad con el medio ambiente e impacto en el desarrollo social de las comunidades. Finalmente, se observa el interés de las empresas en utilizar estándares como la AS 8000 y la ISO 26000 para apoyar la implementación de la responsabilidad social y la logística inversa; de allí la importancia de promover su conocimiento y posterior implementación en el sector empresarial.

\section{CONCLUSIONES}

- La logística inversa es un proceso dentro de la cadena de suministro que permite establecer estrategias para gestionar adecuadamente los retornos, el reciclaje, la remanufactura o disposición de los productos; de allí la importancia de una adecuada gestión de este proceso debido a que influye en la reducción de impactos ambientales y recuperación del valor económico.

- La RSE se enfoca a desarrollar procesos empresariales que generen beneficios en el ámbito económico, social, ético y ambiental a los consumidores y comunidad afectada por las operaciones de la empresa y su cadena 
de suministro sin afectar su rentabilidad ni posicionamiento en el mercado.

- La planeación, implementación y control de estrategias conjuntas de logística inversa y RSE pueden generar beneficios ampliados en la cadena de suministro, ya que se generan prácticas amigables con el medio ambiente y aumento potencial de la rentabilidad de la empresa, y se desarrolla un enfoque integral que crea beneficios en la comunidad, los empleados y los clientes en aspectos éticos, de salud y seguridad, derechos humanos, responsabilidad financiera, implementación de estándares internacionales como AS8000 e ISO 26000, entre otros aspectos.

- Del estudio, se identifica un bajo nivel de utilización de esta práctica con 30\% de las empresas y un interés de $80 \%$ en implementarla, debido a los impactos potenciales en la productividad, medio ambiente y beneficios en la comunidad. Además se identifica el desconocimiento del impacto y las características de la logística inversa como práctica de la responsabilidad social en el estudio exploratorio, lo cual puede convertirse en una oportunidad para desarrollar programas de promoción e implementación teniendo en cuenta los beneficios potenciales descritos que puede tener este enfoque.

- Como trabajos futuros se considera el diseño de una metodología que facilite la implementación de la logística inversa y la RSE, incluyendo el uso de técnicas de modelamiento y gestión que permitan medir los impactos y los beneficios en lo económico, social y demás principios del RSE.

\section{BIBLIOGRAFÍA}

Barker, T.J.;Zabinsky,Z. B. (2008). "Reverselogistics network design: a conceptual framework for decision making", in: International Journal of Sustainable Engineering, 1 (4), p. 250.

Carroll, A. B. (1999). "Corporate social responsability", in: Business \& Society, 38 (3), 268.

Carter, C. R. \& Jennings, M. (2002). "Logistics social responsibility: an integrative framework", in: Journal of Business Logistics, 23 (1), 145-180.

Carter, C. R. \& Rogers, D. S. (2008). "A framework of sustainable supply chain management: moving toward new theory", in: International Journal of Physical Distribution \& Logistics Management, 38 (5), 360-387.

Ciliberti, F.; Potrandolfo, P. \& Scozzi, B. (2008). "Integrating Corporate Social Responsibility in the Management of Supply Chain", in: Journal of cleaner Production, 16 (16), 1679-1688.

Dyckhoff, H.; Lackes, R. \& Reese, J. (2004). Supply chain management and reverse logistics. Berlin: Springer, 22.
Lin, W.T.; Lee, H.C. \& Lee, Y.H. (2009). "A decision model for reverse logistics service providers in determining robust optimal processing quantities of returned products", in: Journal of the Chinese Institute of Industrial Engineers, 26 (5), 397.

Mentzer, J. T. (2001). Supply chain management. London: SAGE.

Murphy, P. (2002). Socially responsible logistics: an exploratory study. United States: American Society of Transportation and Logistics, 52.

Vicarli (2009). Logística y transporte. Consultora Viclarli. Disponible en: http://www.vicarli.es/ es/logistica/inversa/ [citado 10 de noviembre de 2010].

Terrado, A. (2007). La cadena de suministro, Argentina: Monografías, 12.

Vellojín, L.; Meza, J.; Amaya, R. (2008). "Logística Inversa: una herramienta de apoyo a la competitividad de las organizaciones", en: Ingeniería y Desarrollo, 20 (1), 184-202. 